\title{
Algumas Discussões Adicionais sobre o Método de Determinação de $\rho$, a \\ Razão entre a Parte Real e a Parte Imaginária da Amplitude de Espalhamento \\ Hadrônico Elástico na Direção para Frente, a Altas Energias
}

\author{
Some Additional Comments about the Determination of $\rho$, the Ratio \\ Between the Real Part and the Imaginary Part of the Forward Elastic \\ Amplitude, in Hadron Collisions at High Energies
}

Antonio Braz de Pádua ${ }^{1}$; Cléia Guiotti de Pádua²

Resumo

O método correto de determinação de $\rho$, a razão entre as partes real e imaginária da amplitude de espalhamento elástico na direção para frente, nas colisões hadrônicas a altas energias, permanece até hoje uma questão em aberto. Neste trabalho, rediscutimos este problema e propormos duas parametrizações alternativas para ajuste dos dados experimentais de $\mathrm{d} \sigma / \mathrm{dt}$ na região da Interferência Coulombiana - Hadrônica e, como conseqüência, a obtenção do valor de $\rho$. Nossas propostas foram testadas com os dados obtidos na polêmica experiência realizada no CERN em 1986 pela colaboração UA4/1 para a colisão $\bar{p} p$ a $\sqrt{\mathrm{s}}=546 \mathrm{GeV}$. A partir desses ajustes, recalculamos a grandeza $\rho$ e encontramos valores bem menores do que o valor original de $0.24 \pm 0.04$, ou seja, $0.197 \pm 0.020$ e $0.189 \pm 0.030$.

Palavras-chave: Espalhamento Elástico. Altas Energias. Próton. Antipróton. Amplitude de Espalhamento Elástico. Seção de Choque Diferencial Elástica. Pequenos Momentos Transferidos. Região de Coulomb. Região da Interferência Coulombiana. Região Hadrônica.

\begin{abstract}
The correct method of determination of $\rho$, the ratio between the real and imaginary part of the elastic scattering amplitude in the forward direction, in the hadronic collisions at high energy, remains as an is still considered an open subject up till now. In this work we come back to discuss this problem and present two alternative fits for adjustment of the experimental data of $\mathrm{d} \sigma / \mathrm{dt}$ in the Coulomb - Hadronic Interference region are presented, consequently obtaining as well as the value of $\rho$. Our proposals were tested with the data obtained in the polemic experiment accomplished in CERN in 1986 by the UA4/1 collaboration for the $\overline{\mathrm{p}} \mathrm{p}$ collision at $\sqrt{\mathrm{s}}=546 \mathrm{GeV}$. Using these same data, we calculated the variable $\rho$ again and we found values much smaller than the original value of $0.24 \pm 0.04$, namely, $0.197 \pm 0.020$ and $0.189 \pm 0.030$.

Key words: Elastic Scattering, High Energies, Proton, Antiproton, Elastic Scattering Amplitude, Elastic Differential Cross Section. Small Momentum Transfer, Coulomb Region, Coulomb Interference Region, Hadronic Region.
\end{abstract}

\footnotetext{
1 Doutor em Física, Professor Associado C, Departamento de Física, Centro de Ciências Exatas, Universidade Estadual de Londrina. E-mail: padua@uel.br

${ }^{2}$ Mestre em Física, Professor Adjunto D, Departamento de Física, Centro de Ciências Exatas, Universidade Estadual de Londrina. E-mail: guiotti@uel.br
} 


\section{Introdução}

$\mathrm{Na}$ área da Física de Altas Energias, sabe-se que uma determinação experimental precisa e confiável da grandeza $\rho$, definida como a razão entre a parte real e a parte imaginária da amplitude de espalhamento elástico na direção para frente, é fundamental para a compreensão segura de alguns aspectos básicos das colisões hádron - hádron, neste regime de energia.

Até 1985, alguns grupos experimentais do acelerador ISR do CERN (AMOS et al., 1985), utilizando-se de métodos convencionais, fizeram uma série de medidas desta grandeza para as colisões pp e $\bar{p}$ p no intervalo de energias $23 \leq \sqrt{\mathrm{s}} \leq 62 \mathrm{GeV}$. Sem muito questionamento, estas medidas sempre foram consideradas relativamente precisas e confiáveis. Em 1986, com o objetivo de se buscar por informações em energias cada vez mais altas, a Colaboração UA4/1 (BERNARD et al., 1987) apresentou para a colisão $\overline{\mathrm{p}} \mathrm{p}$, realizada no S $\overline{\mathrm{p}} \mathrm{pS}$ COLLIDER do CERN a $\sqrt{\mathrm{s}}=546 \mathrm{GeV}$, o seguinte resultado: $\rho=0.24 \pm 0.04$. Esse dado experimental causou uma verdadeira excitação na comunidade científica envolvida com a Física de Altas Energias, pois ele estava muito acima do valor estimado pela tendência natural dos dados provenientes do ISR do CERN e, também, era muito maior que o valor previsto pela maioria dos modelos teóricos existentes na época. Desde a sua divulgação (a nosso ver, precipitada), ele causou grandes preocupações e reacendeu novas discussões em torno da grandeza $\rho$.

A determinação correta (ou pelo menos mais confiável) de $\rho$ ainda continua uma questão em aberto e requer um pouco mais de cuidado e investigação. Essa observação é pertinente, mesmo considerando uma medida posterior mais cuidadosa, realizada pela Colaboração UA4/2 (AUGIER et al., 1993), para esta colisão e nesta energia. Por meio dela, foi obtido um valor bem mais baixo (qual seja, $\rho \overline{\mathrm{p}} \mathrm{p}=0.135 \pm 0.020)$. Também cabe levar em conta a colisão $\overline{\mathrm{p}} \mathrm{p}$, mas agora sob a responsabilidade da
Colaboração E - 711 (AMOS et al., 1992), no FERMILAB TEVATRON a $\sqrt{\mathrm{s}}=1800 \mathrm{GeV}$, na qual se encontrou o valor $\rho_{\overline{\mathrm{p}} \mathrm{p}}=0.140 \pm 0.069$.

Neste artigo, discutimos os principais problemas existentes na determinação experimental de $\rho$ e sugerimos duas maneiras alternativas de realizá-la de um modo independente de modelo teórico. Mais precisamente, propomos duas expressões analíticas empíricas para amplitude de espalhamento hadrônico elástico e, a partir delas, ajustamos numericamente os dados experimentais da seção de choque diferencial elástica ( $\mathrm{d} \sigma / \mathrm{dt}$ ) num amplo intervalo de pequenos momentos transferidos $(-t)$. Este intervalo compreende as regiões dos espalhamentos Coulombiano (ou de Coulomb), da Interferência Coulombiano - Hadrônico e início do Hadrônico. Com estas duas propostas, conseguimos ajustar melhor os dados de da Colaboração UA4/1, com um valor de $\rho$ menor que 0.24 . Salientamos que nossas propostas não se tratam de novos modelos, mas apenas de formas mais cuidadosas para parametrizar os dados experimentais de na região de momentos transferidos muito pequenos.

Gostaríamos de ter testado nossas idéias com os dados da colisão $\overline{\mathrm{p}} \mathrm{p}$ da Colaboração UA4/2 a $\sqrt{\mathrm{s}}=546 \mathrm{GeV}($ AUGIER et al., 1993) e também, com aqueles a $\sqrt{\mathrm{s}}=1800 \mathrm{GeV}$ (AMOS et al., 1992), mas infelizmente eles não estão disponíveis na unidade de $\left[\mathrm{mb} /(\mathrm{GeV} / \mathrm{c})^{2}\right]$. Eles precisariam ser normalizados, de modo que esse procedimento não será discutido neste trabalho.

O presente artigo está organizado da seguinte maneira: na seção 2, fazemos uma breve descrição do método convencional de determinação experimental de $\rho$, na seção 3 , discutimos os principais problemas e dificuldades apresentadas neste procedimento e, na seção 4, descrevemos nossos métodos de ajustes e apresentamos os resultados obtidos. As observações finais e as conclusões estão na seção 5 . 


\section{O Método Convencional}

Num experimento de colisão para se medir a seção de choque diferencial elástica, a quantidade real medida é a taxa de contagem de eventos $\Delta \mathrm{N}(\mathrm{t})$, ou seja, o número de eventos/segundo/ $\Delta \mathrm{N}(\mathrm{t})$ registrados no detector, onde $\Delta \mathrm{N}(\mathrm{t})$ é um pequeno intervalo de momento transferido ao redor de $-t$, fazendo-se naturalmente as devidas correções. Para se obter $\mathrm{d} \sigma / \mathrm{dt}$, é necessárip̄pormalizar esta taxa da seguinte forma:

$$
\Delta \mathrm{N}(\mathrm{t})=\mathrm{L} \frac{\mathrm{d} \sigma}{\mathrm{dt}}
$$

onde L é o fator de normalização. Para um acelerador de feixes colidentes, Lé a luminosidade.

Dessa forma, os dados experimentais da seção de choque diferencial elástica, expressos em geral por $\left[\mathrm{mb} /(\mathrm{GeV} / \mathrm{c})^{2}\right]$, são dados por:

$$
\frac{\mathrm{d} \sigma}{\mathrm{dt}}=\frac{1}{\mathrm{~L}} \Delta \mathrm{N}(\mathrm{t}) .
$$

Para determinar $\rho$, fazem-se medidas $\mathrm{de} d \sigma / \mathrm{dt}$ para $-t$ muito pequeno, mais exatamente na região da Interferência Coulombiana - Hadrônica. Esta região se estende aproximadamente ao longo do intervalo $0.001 \leq-\mathrm{t} \leq 0.02(\mathrm{GeV} / \mathrm{c})^{2}$. Nesta região, é dada por:

$$
\frac{\mathrm{d} \sigma}{\mathrm{dt}}=\pi\left|\mathrm{F}_{\mathrm{c}}(\mathrm{s}, \mathrm{t}) \mathrm{e}^{\mp i \alpha \phi(\mathrm{t})}+\mathrm{F}_{\mathrm{h}}(\mathrm{s}, \mathrm{t})\right|^{2}
$$

onde $\mathrm{F}_{\mathrm{c}}(\mathrm{s}, \mathrm{t})$ é conhecida como amplitude de Coulomb (ou Coulombiana), $F_{h}(s, t)$ é a amplitude hadrônica (nuclear, ou forte) e $\exp [\mp i \alpha \phi(t)]$ é o fator de fase que reflete a distorção das amplitudes puras $\mathrm{F}_{\mathrm{c}}(\mathrm{s}, \mathrm{t})$ e $F_{h}(s, t)$, devida a presença simultânea dos espalhamentos Coulombiano e Hadrônico.

A amplitude de Coulomb é dada por:

$$
F_{c}(t)=\frac{ \pm 2 \alpha^{2} G^{2}(t)}{-t}
$$

onde $\alpha \cong 1 / 137$ é a constante de estrutura fina e $\mathrm{G}(\mathrm{t})$ o fator de forma eletromagnético do próton, representado normalmente pela expressão de dipolo:

$$
G(t)=\frac{1}{\left(1+\frac{-t}{0.71}\right)^{2}}
$$

A fase Coulombiana - Hadrônica $\phi(t)$ é dada por (BLOCK; CAHN, 1985):

$$
\phi(t)=\ln \left(\frac{0.08}{-t}-0.577\right)
$$

A amplitude de espalhamento hadrônico elástico é, em geral, uma função complexa, ou seja:

$$
\mathrm{F}_{\mathrm{h}}(\mathrm{s}, \mathrm{t})=\operatorname{Re} \mathrm{F}_{\mathrm{h}}(\mathrm{s}, \mathrm{t})+\mathrm{i} \operatorname{Im} \mathrm{F}_{\mathrm{h}}(\mathrm{s}, \mathrm{t})
$$

$\mathrm{Na}$ direção para frente $(-\mathrm{t}=0)$, usando-se o Teorema Óptico, a equação (7), pode ser escrita como:

$$
\mathrm{F}_{\mathrm{h}}(\mathrm{s}, 0)=\frac{\sigma_{\mathrm{tot}}(\mathrm{s})}{4 \pi}[\rho(\mathrm{s}, 0)+\mathrm{i}]
$$

onde $\rho(\mathrm{s}, 0) \equiv \rho=\operatorname{ReF}_{\mathrm{h}}(\mathrm{s}, 0) / \operatorname{ImF}_{\mathrm{h}}(\mathrm{s}, 0)$.

Tradicionalmente, a amplitude de espalhamento hadrônico elástico $F_{h}(s, t)$, para pequenos momentos transferidos, tem sido representada por uma única exponencial, da seguinte forma:

$$
F_{h}(s, t)=\frac{\sigma_{\text {tot }}(s)}{4 \pi}(\rho+i) e^{\frac{B}{2} t}
$$

onde, B é o parâmetro de inclinação ("slope").

É exatamente neste procedimento que reside um dos principais problemas na determinação de $\rho$.

Pela substituição das relações (4) e (9) em (3), obtemos a expressão completa para $\mathrm{d} \sigma / \mathrm{dt}$ em uma região de -t muito pequeno e para a condição $\alpha \phi<<1$, qual seja:

$\frac{\mathrm{d} \sigma}{\mathrm{dt}}=\pi \frac{4 \alpha^{2} \mathrm{G}^{2}(\mathrm{t})}{(-\mathrm{t})^{2}} \pm \pi \frac{\sigma_{\mathrm{tot}} \alpha(\rho \mp \alpha \phi) \mathrm{G}^{2}(\mathrm{t}) \mathrm{e}^{\frac{\mathrm{B}}{2}}}{-\mathrm{t}}+\pi \frac{\left(1+\rho^{2}\right) \sigma_{\mathrm{tote}}^{2} \mathrm{e}^{\mathrm{Bt}}}{(4 \pi)^{2}}$,

onde os sinais superiores e inferiores se referem às colisões $\bar{p} p$ e pp, respectivamente. 
Os três termos na Eq. 10 descrevem as contribuições de Coulomb, Interferência Coulombiana - Hadrônica e Hadrônica para do/dt, respectivamente. A interferência máxima entre os espalhamentos Coulombiano e Hadrônico ocorre em $-\mathrm{t}_{\text {int }} \sim 8 \pi \alpha / \sigma_{\text {tot }}$. O correspondente ângulo de espalhamento no sistema do centro de massa é dado por $\theta_{\text {int }}=\sqrt{-\mathrm{t}_{\text {int }}} / \mathrm{k} \quad(\mathrm{k} \quad$ é $\quad \mathrm{o}$ momento $\mathrm{da}$ partícula incidente). A Tabela 1 mostra os valores de $-t_{\text {int }}$ e $\theta_{\text {int }}$ da Interferência Coulombiana Hadrônica para o espalhamento elástico $\bar{p}$ das energias do ISR CERN até o SSC - BNL (este acelerador não foi construído até hoje). A seção de choque total a $\sqrt{\mathrm{s}}=40000 \mathrm{GeV}=40 \mathrm{TeV}$ foi estimada em $\sim 127 \mathrm{mb}$ (DIAS DE DEUS; PÁDUA, 1993).

Tabela 1. Valores de $-t_{\text {int }}$ e $\theta_{\text {int }}$, para os quais ocorre o máximo da Interferência Coulombiana - Hadrônica no espalhamento elástico .

\begin{tabular}{|l|l|l|l|}
\hline$\sqrt{\mathrm{s}}(\mathrm{GeV})$ & \multicolumn{1}{|c|}{ ACELERADOR } & $-\mathrm{t}_{\text {int }}(\mathrm{GeV} / \mathrm{c})^{2}$ & $\theta_{\text {int }}(\mathrm{mrd})$ \\
& & & \\
\hline 23.5 & ISR - CERN & 0.0017 & 3.5 \\
\hline 30.4 & ISR - CERN & 0.0016 & 2.6 \\
\hline 52.6 & ISR - CERN & 0.0016 & 1.5 \\
\hline 62.3 & ISR - CERN & 0.0016 & 1.3 \\
\hline 546 & $\mathrm{~s} \overline{\mathrm{p}} \mathrm{p}$ - - COLLIDER - CERN & 0.00115 & 0.12 \\
& TEVATRON - FERMILAB & 0.00098 & 0.035 \\
\hline 1800 & SSC - BNL & 0.00056 & 0.0012 \\
\hline 40000 & \multicolumn{2}{l}{} \\
\hline
\end{tabular}

Usando-se as equações (2), (3) e (8), podemos escrever agora:

$$
\sigma_{\text {tot }}^{2}\left(1+\rho^{2}\right)=16 \pi \frac{\Delta N(0)}{L},
$$

onde $\Delta \mathrm{N}(0)$ é a taxa de contagem hadrônica em $-t=0$. Esta expressão, em geral, é usada para fixar a normalização absoluta. Podemos fazer isto de duas maneiras: (1) Primeiramente, parametrizamos os dados de $\mathrm{d} \sigma / \mathrm{dt}$ na região de Coulomb pela Eq. 4 e, depois, determinarmos L através da Eq. 1. (2) Sabendo-se que $\sigma_{\text {tot }}=\mathrm{N}_{\text {tot }} / \mathrm{L}$, onde $\mathrm{N}_{\text {tot }}$ é a taxa de contagem total devido a um espalhamento qualquer, podemos escrever a Eq. 11 da seguinte forma:

$$
\sigma_{\text {tot }}\left(1+\rho^{2}\right)=\frac{16 \pi \Delta \mathrm{N}(0)}{\mathrm{N}_{\text {tot }}} \text {. }
$$

Devemos notar que, em ambos os casos, é necessário obter $\Delta \mathrm{N}(0)$, a taxa de contagem hadrônica extrapolada para $-\mathrm{t}=0$. Normalmente, $\mathrm{a}$ extrapolação é feita usando-se uma única exponencial para representar os dados de seção de choque diferencial elástica a baixos momentos transferidos. Como já manifestamos, ao nosso ver, isto não é um procedimento muito correto.

A combinação $\sigma_{\text {tot }}\left(1+\rho^{2}\right)$ foi medida por Bozzo et al. (1984) para a colisão de $\overline{\mathrm{p}} \mathrm{p}$ a $\sqrt{\mathrm{s}}=546 \mathrm{GeV}$. O resultado obtido foi o seguinte:

$$
\sigma_{\text {tot }}\left(1+\rho^{2}\right)=63.3 \pm 1.5 \mathrm{mb}
$$

\section{Problemas e Dificuldades no Cálculo de $\rho$}

Leader (1987) fez no "Second Blois Workshop" uma excelente discussão sobre os problemas de determinação da grandeza $\rho$. Apoiando-se nas observações de Leader e incorporando-se os resultados de trabalhos que desenvolvemos nos últimos anos (PÁDUA; PÁDUA, 2002), podemos dizer que os problemas no cálculo de $\rho$ têm três origens básicas: (i) dificuldades de se medir a seção de choque diferencial elástica $\mathrm{d} \sigma / \mathrm{dt}$ em -t muito pequeno; (ii) utilização de expressões analíticas inadequadas para a amplitude de espalhamento hadrônico elástico, (iii) maneira de realizar os ajustes numéricos. A seguir vamos discuti-las com alguns pormenores!

(i) Dificuldades de se medir a seção de choque diferencial elástica $\mathrm{d} \sigma / \mathrm{dt}$ em -t muito pequeno.

Enquanto a idéia do método experimental de medição de $\rho$ é, em princípio, direta, a necessidade de detectar eventos elásticos em ângulos muito pequenos apresenta complicações técnicas sérias. Esse fato não tem permitido que, em quase todos os experimentos, haja penetração na região da 
Interferência Coulombiana - Hadrônica, quando se procura medir $\mathrm{d} \sigma / \mathrm{dt}$. Isso tem sido mais crítico com o aumento da energia. Por exemplo, nos casos dos experimentos realizados com a colisão de $\overline{\mathrm{p}} \mathrm{p}$, pela Colaboração UA4/1 (BERNARD et al., 1987) a $\sqrt{\mathrm{s}}=546 \mathrm{GeV}$, as medidas foram, até $-\mathrm{t}=0.00225(\mathrm{GeV} / \mathrm{c})^{2}$; pela Colaboração UA4/2 (AUGIER et al., 1993) na mesma energia, até $-\mathrm{t}=0.001(\mathrm{GeV} / \mathrm{c})^{2}$, por Amos et al. (1985) nas energias de $\sqrt{\mathrm{s}}=30.4,52.6$ e $62.3 \mathrm{GeV}$, foram até os valores $-t=0.00067(\mathrm{GeV} / \mathrm{c})^{2}, \quad-t=0.00097(\mathrm{GeV} / \mathrm{c})^{2} \mathrm{e}$ $-\mathrm{t}=0.00632(\mathrm{GeV} / \mathrm{c})^{2}$, respectivamente e, finalmente, pela Colaboração E - 710 (AMOS et al., 1992) a $\sqrt{\mathrm{s}}=1800 \mathrm{Ge}$, atingiu-se $-\mathrm{t}=0.00095(\mathrm{GeV} / \mathrm{c})^{2}$. Podemos verificar pela comparação com os dados da Tabela 1 que nenhum destes experimentos alcançou por inteiro $\left(-t<-t_{\text {int }}\right)$ a região da Interferência Coulombiana - Hadrônica. Somente nas energias de $30.7,52.6$ e $1800 \mathrm{GeV}$ as medidas foram até por volta $\mathrm{de}-\mathrm{t}_{\text {int }}$. Acreditamos que este seja o primeiro grande problema na determinação de $\rho$, pois como veremos na próxima seção, o segundo termo da Eq. 10 (valores na Tabela 2) é muito significativo para $-\mathrm{t} \leq 0.002(\mathrm{GeV} / \mathrm{c})^{2}$.

Outro problema relacionado com o experimento é a grande flutuação na distribuição de dados. $\mathrm{Na}$ reação $\overline{\mathrm{p}} \mathrm{p}$, isso é verificado nas energias de $\sqrt{\mathrm{s}}=62.3 \mathrm{GeV}($ AMOS et al., 1985) e $\sqrt{\mathrm{s}}=546 \mathrm{GeV}$ (BERNARD et al., 1987). No caso do experimento da Colaboração UA4/1 (BERNARD et al., 1987), nosso ponto de referência principal no presente trabalho, este problema é muito sério. O comportamento dos pontos experimentais, principalmente dos três primeiros, afeta fortemente a extrapolação a baixos valores de $-\mathrm{t}$, porque eles são os mais relevantes para se penetrar na região da Interferência Coulombiana - Hadrônica (DIAS DE DEUS, 1987).

(ii) A utilização de expressões analíticas inadequadas para a amplitude de espalhamento hadrônico.

A parametrização convencional da amplitude de espalhamento hadrônico como uma única exponencial, com um parâmetro de inclinação de sua parte real igual ao de sua parte imaginária e ainda independente do momento transferido, não está correto nem mesmo em regiões de $-\mathrm{t}$ muito pequeno. Em análises independentes de modelo teórico (PÁDUA et al., 1991; PÁDUA; PÁDUA, 2002) da colisão $\bar{p} p$ até $\sqrt{\mathrm{s}}=546 \mathrm{GeV}$, onde os dados experimentais de $\mathrm{d} \sigma / \mathrm{dt}$ foram ajustados, tomando-se:

$$
\begin{gathered}
\operatorname{Im} F_{h}(s, t)=\sum_{i=1}^{n} \alpha_{i} e^{\beta_{i} t} \\
\operatorname{ReF}_{h}(s, t)=\sum_{i=1}^{n} \alpha_{i}\left[1+\beta_{i} t\right] e^{\beta_{i} t},
\end{gathered}
$$

verificamos que o parâmetro de inclinação da parte real da amplitude de espalhamento elástico $\left(B_{R}\right)$ na direção para frente $(-t=0)$ é maior que o parâmetro de sua parte imaginária $\left(\mathrm{B}_{\mathrm{I}}\right)$ no mesmo ponto. Mais precisamente, encontramos que: $\mathrm{B}_{\mathrm{R}}(0) \cong 2 \mathrm{~B}_{\mathrm{I}}(0)$. Considerando este fato, os resultados (mostrados na próxima seção) apresentam uma mudança significativa no valor de $\rho$, em relação ao obtido pela Colaboração UA4/1 (BERNARD et al., 1987) para a reação $\overline{\mathrm{p} p}$ a $\sqrt{\mathrm{s}}=546 \mathrm{GeV}$.

A respeito do parâmetro de inclinação ser independente do momento transferido $-\mathrm{t}$ também não é um fato verdadeiro. Algumas análises, entre elas podemos citar, as das referências 8, 9, 10 e 11 (DIAS DE DEUS; PÁDUA, 1993; LEADER, 1987; PÁDUA et al., 1991; PÁDUA; PÁDUA, 2002), mostram que o parâmetro de inclinação cresce quando $-\mathrm{t}$ decresce. Devemos considerar também que, para cada valor de $-\mathrm{t}, \quad \mathrm{o}$ parâmetro de inclinação cresce com o aumento da energia e esta variação é aproximadamente proporcional a $\sigma_{\text {tot }}$ (DIAS DE DEUS, 1987). Uma maneira de implementar isso seria usar na Eq. 10 uma expressão para $\mathrm{B}$ em função de $\mathrm{s}$ e de $\mathrm{t}$, tal 
como $\mathrm{B}(\mathrm{s}, \mathrm{t}) \cong \sigma_{\text {tot }}(\mathrm{s})(-\mathrm{t})+\mathrm{C}(\mathrm{s})$. Se estamos interessados apenas no cálculo de $\rho$ e não desejamos um valor de $\mathrm{B}$ ou nem trabalhar com um parâmetro de inclinação definido localmente, nós podemos usar uma expressão mais flexível para amplitude hadrônica do que a tradicional exponencial simples, por exemplo, uma soma de exponenciais em - t (como a Eq. 14). Este tipo de função se ajustaria melhor às pequenas mudanças dos dados em $-\mathrm{t}$ muito pequeno e extrapolaria para ponto ótico, $-\mathrm{t}=0$, com maior precisão. A análise da reação $\bar{p} p$ para altas energias (PADUA et al., 1991; PÁDUA; PÁDUA, 2002) mostra claramente que a amplitude de espalhamento elástico no espaço do parâmetro de impacto não é uma gaussiana pura, mas tem uma forma bem próxima de uma combinação de gaussianas. Na seção seguinte, nós usaremos a amplitude hadrônica como uma soma de exponenciais em - t para calcular $\rho$, por meio dos dados experimentais de $\mathrm{d} \sigma / \mathrm{dt}$ da Colaboração UA4/1 (BERNARD et al., 1987).

(iii) A maneira de realizar os ajustes numéricos.

Depois da medida de $\mathrm{d} \sigma / \mathrm{dt}$ a $-\mathrm{t}$ pequeno, podemos usar a Eq. 10 para realizar o ajuste. Em princípio, esta equação tem três parâmetros livres, $\sigma_{\text {tot }}, \rho$ e B. Poderia ter um quarto, se os dados experimentais estivessem na forma de $\Delta \mathrm{N}(\mathrm{t})$ e este seria a luminosidade $\mathrm{L}$.

Aparentemente, está tudo certo, mas, em geral, ocorre mais um problema que pode afetar o valor de $\rho$, ou seja, nem todos os experimentos fazem o ajuste dos próprios dados deixando $\sigma_{\text {tot }}, \rho$ e B como parâmetros livres. Para a colisão $\bar{p} p$, por exemplo, Amos et al. (1985) determinam $\sigma_{\text {tot }}$ para todas as energias que são estudadas, isto é, 30.4, 52.6 e $62.3 \mathrm{GeV}$. No entanto, $\rho$ não é calculada a $62.3 \mathrm{GeV}$, porque seus dados cobrem somente uma parte da região da Interferência Coulombiana - Hadrônica e $\mathrm{B}$ também não é determinado a $30.4 \mathrm{GeV}$, porque os dados não estendem até - $t$ suficientemente grandes para que possam permitir uma medida confiável do parâmetro de inclinação hadrônico. Em ambos os casos, são usados parâmetros de outros trabalhos como "input" dos ajustes. Somente a $\sqrt{\mathrm{s}}=52.6 \mathrm{GeV}$ são calculados $\sigma_{\text {tot }}, \rho$ e $\mathrm{B}$ simultaneamente; no TEVATRON a $\sqrt{\mathrm{s}}=1800 \mathrm{GeV}$ (AMOS et al., 1992), é feito um ajuste completo e se calculam todos os três parâmetros e, finalmente, é no experimento da Colaboração UA4/1 (BERNARD et al., 1987), a $\sqrt{\mathrm{s}}=546 \mathrm{GeV}$, que acontece a maioria dos problemas. Primeiro, eles realizam um ajuste num intervalo amplo de $-\mathrm{t}$, deixando $\rho$ e $\mathrm{B}$ como parâmetros livres. Os resultados encontrados foram $\mathrm{B}=15.5 \pm 0.8(\mathrm{GeV} / \mathrm{c})^{-2}$ e $\rho=0.24 \pm 0.04$. Posteriormente, o ajuste foi realizado num intervalo menor de $-\mathrm{t}$, fixando $\rho$ em 0.23 e obtiveram $\mathrm{B}=15.3 \pm 0.3(\mathrm{GeV} / \mathrm{c})^{-2}$. Por fim, $\mathrm{B}$ foi fixado no valor anteriore $\rho$, juntamente com $\mathrm{L}$, foram deixados como parâmetros livres. O resultado deste ajuste final foi $\rho=0.238 \pm 0.024$. Em todos os ajustes, a normalização absoluta foi fixada pela Eq. 13, obtida de outros experimentos (BOZZO et al., 1984). O resultado final, considerando também os erros estatísticos, foi exatamente o controverso valor $0.24+0.04$.

\section{Parametrizações Alternativas para a Determinação de $\rho$.}

Nossas propostas visando a uma determinação mais adequada da quantidade $\rho$, são as seguintes:

$\left.1^{a}\right)$ Apoiando-se no fato de que $B_{R}$ é diferente do $\mathrm{B}_{\mathrm{I}}$, propomos representar a amplitude do espalhamento hadrônico elástico através da seguinte expressão:

$$
F_{h}(s, t)=\frac{\sigma_{\text {tot }}}{4 \pi}\left[\rho e^{\frac{B_{R} t}{2}}+i e^{\frac{B_{I} t}{2}}\right]
$$

Assim, substituindo-se (4) e (16) em (3), com a condição de que $\alpha \phi<<1$, obtemos:

$$
\begin{aligned}
& \frac{d \sigma}{d t}=N\left\{\frac{4 \alpha^{2} G^{4}(t)}{(-t)^{2}} \pm \frac{\alpha G^{2}(t) \sigma_{t o t}}{-t \pi}\left[\rho e^{\frac{B_{R} t}{2}} \mp \alpha \varphi e^{\frac{B_{I} t}{2}}\right]+\right. \\
& \left.\frac{\sigma_{\text {tot }}^{2}}{(4 \pi)^{2}}\left[\rho^{2} e^{B_{R} t}+e^{B_{I} t}\right]\right\}
\end{aligned}
$$


onde os sinais superiores e inferiores se referem, respectivamente, às colisões $\overline{\mathrm{p}} \mathrm{p}$ e pp e, $\mathrm{N}$ é qualquer constante multiplicada por $\pi$. Usamos $N$ como um parâmetro livre para evitar qualquer problema de normalização.

$\mathrm{Na}$ Eq. 17, os três termos representam as seções de choque diferenciais elásticas de Coulomb $\left(\mathrm{d} \sigma_{\mathrm{c}} / \mathrm{dt}\right)$, da Interferência Coulombiana-Hadrônica $\left(\mathrm{d} \sigma_{\mathrm{ch}} / \mathrm{dt}\right)$ e Hadrônica $\left(\mathrm{d} \sigma_{\mathrm{h}} / \mathrm{dt}\right)$, respectivamente.

Os resultados obtidos ajustando-se todos os dados da Colaboração UA4/1 (BERNARD et al., 1987) por meio da Eq. 17 são os seguintes:

$$
\begin{aligned}
& \mathrm{N}=3.222 \pm 0.032, \quad \sigma_{\text {tot }}=60.01 \pm 0.31 \mathrm{mb}, \\
& \mathrm{B}_{\mathrm{R}}=27.74 \pm 1.43(\mathrm{GeV} / \mathrm{c})^{-2} \\
& \mathrm{~B}_{\mathrm{I}}=14.97 \pm 0.54(\mathrm{GeV} / \mathrm{c})^{-2} \quad \rho=0.197 \pm 0.020 \\
& \chi^{2} / \mathrm{gdl}=1.72
\end{aligned}
$$

A Figura 1 mostra o resultado de nosso melhor ajuste em comparação com os dados experimentais. Nesta figura, a linha contínua representa a equação 17 completa e a linha tracejada apenas a parte da seção de choque diferencial elástica hadrônica.

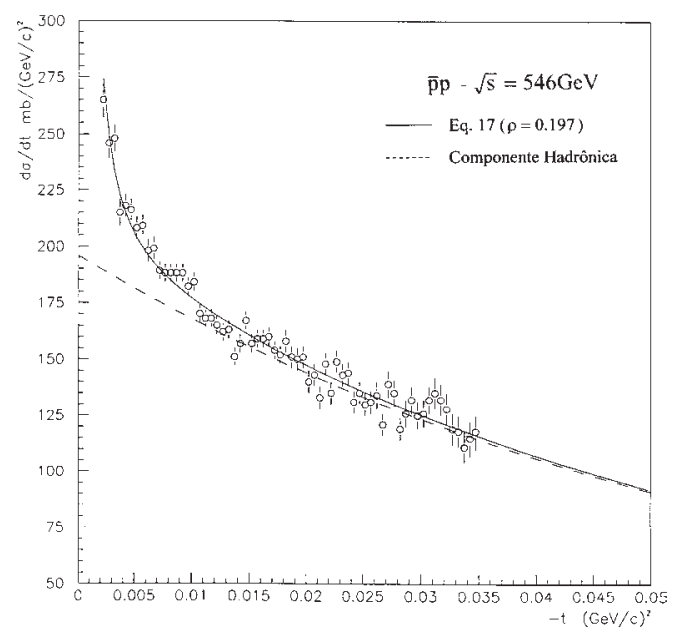

Figura 1. Seção de choque diferencial elástica da colisão $\overline{\mathrm{p}} \mathrm{p}$ a $\sqrt{\mathrm{s}}=546 \mathrm{GeV}$ na região da Interferência Coulombiana - Hadrônica. A linha contínua representa o melhor ajuste feito através da equação 17 completa e a linha tracejada se refere somente à componente hadrônica. Os dados experimentais foram obtidos pela Colaboração UA4/ 1 do CERN (BERNARD et al., 1987).
O ponto óptico calculado pelo ajuste é $(\mathrm{d} \sigma / \mathrm{dt})_{-\mathrm{t}=0}=195.70 \mathrm{mb} /(\mathrm{GeV} / \mathrm{c})^{2}$. Se tomamos $\mathrm{N}=\pi$, este valor cai para $(\mathrm{d} \sigma / \mathrm{dt})_{-\mathrm{t}=0}=190.82 \mathrm{mb} /(\mathrm{GeV} / \mathrm{c})^{2}$. Este último valor é consistente com $(\mathrm{d} \sigma / \mathrm{dt})_{-\mathrm{t}=0}=190.79 \pm 3.41 \mathrm{mb} /(\mathrm{GeV} / \mathrm{c})^{2}$, que foi calculado pela substituição dos novos valores de $\sigma_{\text {tot }} \mathrm{e}$ $\rho$ na Eq. 11. Então, a utilização de $\mathrm{N}$ como um parâmetro livre só faz a curva de ajuste subir ou baixar como um todo e não interfere nos valores de $\sigma_{\text {tot }}$, $\rho, \mathrm{B}_{\mathrm{R} \text { e }} \mathrm{B}_{\mathrm{I}}$.

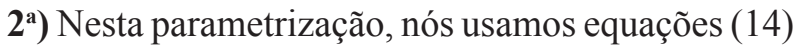
e (15) para representar a amplitude hadrônica (PÁDUA et al., 1991; PÁDUA; PÁDUA, 2002). Assim, temos:

$$
F_{h}(s, t)=\rho \sum_{i=1}^{n} \alpha_{i}\left[1+\beta_{i} t\right] e^{\beta_{i} t}+i \sum_{i=1}^{n} \alpha_{i} e^{\beta_{i} t}
$$

Agora, substituindo-se (4) e (18) em (3), nós obtemos a seguinte expressão completa para $\mathrm{d} \sigma / \mathrm{dt}$ :

$$
\frac{d \sigma}{d t}=N \frac{4 \alpha^{2} G^{4}(t)}{(-t)^{2}}+N\left\{\frac{4 \alpha^{2} G(t) \rho}{-t} \sum_{i=1}^{n} \alpha_{i}\left(1+\beta_{i} t\right)\right.
$$$$
\left.\beta_{\mathrm{i}} \mathrm{t}-\frac{4 \alpha^{2} \phi(\mathrm{t}) \mathrm{G}^{2}(\mathrm{t})}{-\mathrm{t}} \sum_{\mathrm{i}=1}^{\mathrm{n}} \alpha_{\mathrm{i}} \mathrm{e}^{\beta_{\mathrm{i}} \mathrm{t}}\right\}+
$$

$$
\mathrm{N}\left\{\left[\sum_{i=1}^{\mathrm{n}} \alpha_{\mathrm{i}} \mathrm{e}^{\beta_{\mathrm{i}} \mathrm{t}}\right]^{2}+\rho^{2}\left[\sum_{\mathrm{i}=1}^{\mathrm{n}} \alpha_{\mathrm{i}}\left(1+\beta_{\mathrm{i}} \mathrm{t}\right) \mathrm{e}^{\beta_{\mathrm{i}} \mathrm{t}}\right]^{2}\right\}
$$

onde, os três termos são as seções de choque diferenciais elásticas de Coulomb $\left(\mathrm{d} \sigma_{\mathrm{c}} / \mathrm{dt}\right)$, da Interferência Coulombiana - Hadrônica $\left(\mathrm{d} \sigma_{\mathrm{ch}} / \mathrm{dt}\right)$ e Hadrônica $\left(\mathrm{d} \sigma_{\mathrm{h}} / \mathrm{dt}\right)$, respectivamente.

Os resultados obtidos ajustando-se todos os dados experimentais da Colaboração UA4/1 (BERNARD et al., 1987) através da Eq. 19, com $n=2$ são os seguintes:

$$
\beta_{1}=18.600 \pm 1.246(\mathrm{GeV} / \mathrm{c})^{-2}
$$

$\beta_{2}=5.987 \pm 0.788(\mathrm{GeV} / \mathrm{c})^{-2}$ 
$\alpha_{1}=4.130 \pm 0.130\left[\mathrm{mb} /(\mathrm{GeV})^{2}\right]^{1 / 2}, \mathrm{~N}=1.059 \pm 0.017$, $\alpha_{2}=9.696 \pm 0.111\left[\mathrm{mb} /(\mathrm{GeV} / \mathrm{c})^{2}\right]^{1 / 2}, \quad \chi_{\mathrm{e}}^{2} / \mathrm{gdl}=1.75$

A Figura 2 mostra o resultado obtido pelo melhor ajuste em comparação com os dados experimentais. Nesta figura, a linha contínua representa a Eq. 19 completa e a linha tracejada apenas a parte hadrônica desta expressão. Com este ajuste, encontramos um ponto óptico com um valor ligeiramente acima do usual, ou seja, $(\mathrm{d} \sigma / \mathrm{dt})_{-\mathrm{t}=0} \cong 207 \mathrm{mb} /(\mathrm{GeV} / \mathrm{c})^{2}$. Isto corresponde a uma seção de choque total de $\sigma_{\text {tot }} \sim 62 \mathrm{mb}$.

Quando examinamos os resultados apresentados nas figuras 1 e 2 com um pouco mais de cuidado, ou seja, com base numa análise global (considerandose os valores do $\chi^{2}$, os dados mostrados nas diferentes regiões de $\mathrm{d} \sigma / \mathrm{dt}$ da Tabela 2 e os erros da grandeza $\rho$ ), os dados experimentais da seção de choque diferencial elástica em todo intervalo de$\mathrm{t}$, parecem ser igualmente bem representados pelas duas formas de ajustes. As maiores diferenças entre elas, ocorrem na região de $\mathrm{d} \sigma_{\mathrm{ch}} / \mathrm{dt}$.

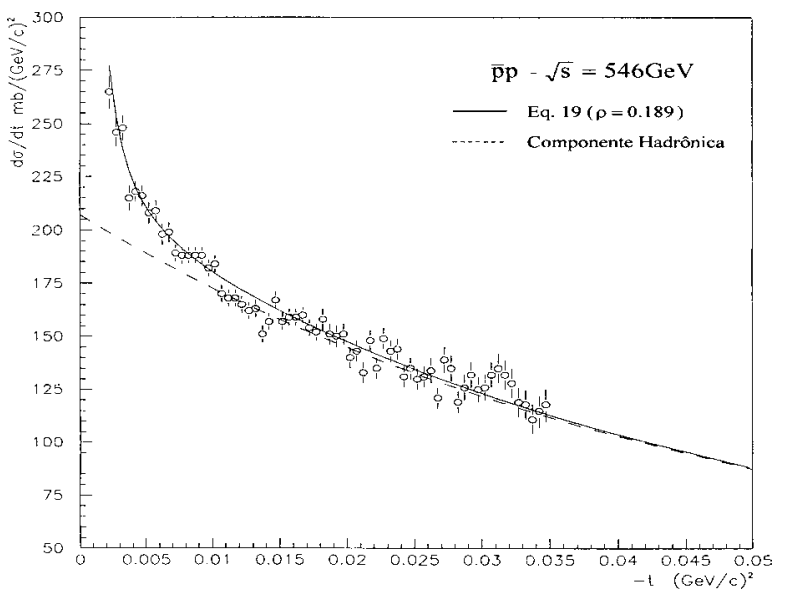

Figura 2. Seção de choque diferencial elástica da colisão $\overline{\mathrm{p}} \mathrm{p}$ a $\sqrt{\mathrm{s}}=546 \mathrm{GeV}$ na região da Interferência Coulombiana - Hadrônica. A linha contínua representa o melhor ajuste feito através da equação 19 completa e a linha tracejada mostra se refere somente à componente hadrônica. Os dados experimentais foram obtidos pela Colaboração UA4/ 1 do CERN (BERNARD et al., 1987).
Na Tabela 2, apresentamos em separado as contribuições de Coulomb, Interferência Coulombiana - Hadrônica e Hadrônica da seção de choque diferencial elástica calculada por meio das equações 10, 17 e 19, para alguns valores característicos de $-t$. Todas as seções de choque diferenciais são expressas em $\mathrm{mb} /(\mathrm{GeV} / \mathrm{c})^{2}$. Esses resultados mostram claramente que as maiores contribuições para $\mathrm{d} \sigma_{\mathrm{ch}} / \mathrm{dt}$ ocorrem para $-\mathrm{t} \leq 0.002(\mathrm{GeV} / \mathrm{c})^{2} \mathrm{e}$ as diferenças mais significativas entre os termos $\mathrm{d} \sigma_{\mathrm{c}} / \mathrm{dt}, \mathrm{d} \sigma_{\mathrm{ch}} / \mathrm{dt}$ e $\mathrm{d} \sigma_{\mathrm{h}} / \mathrm{dt}$, quando são calculados através das equações 10 (modo convencional), 17 $\left(\mathrm{B}_{\mathrm{R}} \neq \mathrm{B}_{\mathrm{I}}\right)$ e $19\left[\mathrm{~F}_{\mathrm{h}}(\mathrm{s}, \mathrm{t})\right)$ como uma soma de exponenciais] respectivamente, são verificadas em $\mathrm{d} \sigma_{\mathrm{ch}} / \mathrm{dt}$.

Tabela 2. Seções de choque diferenciais elásticas de Coulomb, da Interferência Coulombiana - Hadrônica e Hadrônica, calculadas através das equações 10, 17 e 19, respectivamente, para alguns valores característicos de - t Todas as seções de choque são expressas na unidade $\mathrm{mb} /(\mathrm{GeV} / \mathrm{c})^{2}$.

\begin{tabular}{|c|c|c|c|c|c|}
\hline$-\mathrm{t}(\mathrm{GeV} / \mathrm{c})^{2}$ & ajuste & $\mathrm{d} \sigma_{\mathrm{c}} / \mathrm{dt}$ & $\mathrm{d} \sigma_{\mathrm{ch}} / \mathrm{dt}$ & $\mathrm{d} \sigma_{\mathrm{h}} / \mathrm{dt}$ & $\mathrm{d} \sigma_{\text {total }} / \mathrm{d}$ \\
\hline 0.0001 & $\begin{array}{l}\text { Eq. } 10 \\
\text { Eq. } 17 \\
\text { Eq. } 19\end{array}$ & $\begin{array}{l}26046 \\
26712 \\
26956\end{array}$ & $\begin{array}{l}855 \\
682 \\
630\end{array}$ & $\begin{array}{l}194 \\
196 \\
207\end{array}$ & $\begin{array}{l}27095 \\
27590 \\
27793\end{array}$ \\
\hline 0.001 & $\begin{array}{l}\text { Eq. } 10 \\
\text { Eq. } 17 \\
\text { Eq. } 19\end{array}$ & $\begin{array}{l}258 \\
254 \\
267\end{array}$ & $\begin{array}{l}92 \\
74 \\
59\end{array}$ & $\begin{array}{l}192 \\
193 \\
203\end{array}$ & $\begin{array}{l}542 \\
531 \\
529\end{array}$ \\
\hline $\begin{array}{c}0.00115 \\
\left(-t_{\text {int }}\right)\end{array}$ & $\begin{array}{l}\text { Eq. } 10 \\
\text { Eq. } 17 \\
\text { Eq. } 19\end{array}$ & $\begin{array}{l}195 \\
199 \\
206\end{array}$ & $\begin{array}{l}80 \\
65 \\
65\end{array}$ & $\begin{array}{l}191 \\
193 \\
205\end{array}$ & $\begin{array}{l}466 \\
457 \\
476\end{array}$ \\
\hline 0.002 & $\begin{array}{l}\text { Eq. } 10 \\
\text { Eq. } 17 \\
\text { Eq. } 19\end{array}$ & $\begin{array}{l}60 \\
63 \\
64\end{array}$ & $\begin{array}{l}45 \\
37 \\
30 \\
\end{array}$ & \begin{tabular}{|l|}
188 \\
189 \\
200
\end{tabular} & $\begin{array}{l}293 \\
289 \\
294 \\
\end{array}$ \\
\hline 0.01 & $\begin{array}{l}\text { Eq. } 10 \\
\text { Eq. } 17 \\
\text { Eq. } 19\end{array}$ & $\begin{array}{l}2.3 \\
2.4 \\
2.4\end{array}$ & $\begin{array}{l}8.8 \\
6.9 \\
5.3\end{array}$ & $\begin{array}{l}167 \\
168 \\
173\end{array}$ & $\begin{array}{l}178.1 \\
177.3 \\
180.7\end{array}$ \\
\hline 0.03 & $\begin{array}{l}\text { Eq. } 10 \\
\text { Eq. } 17 \\
\text { Eq. } 19\end{array}$ & $\begin{array}{l}0.21 \\
0.21 \\
0.21 \\
\end{array}$ & $\begin{array}{l}2.3 \\
1.6 \\
1.2\end{array}$ & $\begin{array}{l}122 \\
123 \\
122\end{array}$ & $\begin{array}{l}124.5 \\
124.8 \\
123.4 \\
\end{array}$ \\
\hline
\end{tabular}

Observação: Os ajustes dos dados experimentais de seção de choque diferencial elástica através das expressões 17 e 19 foram feitos utilizando-se o método dos mínimos quadrados para funções arbitrárias denominado GRIDLS (PÁDUA et al., 1991).

\section{Discussões Finais e Conclusões}

De modo geral, observamos que, devido a grande importância das grandezas, como $\sigma_{\text {tot }}, \rho$ e B, para a compreensão das colisões hadrônicas a altas energias, é necessário contornar as dificuldades 
técnicas experimentais e obter os dados $d e d \sigma / d t$ com menores flutuações e em intervalos de $-t$ mais adequados. Na determinação de $\rho$, um dos problemas principais tem sido a má qualidade dos dados de $d \sigma / d t$. Se isto não for resolvido, não vale a pena tentar determinar $\rho$. Vejamos, por exemplo, o caso do experimento com a colisão $\bar{p} p$ da Colaboração UA4/1 (BERNARD et al., 1987) a $\sqrt{\mathrm{s}}=546 \mathrm{GeV}$ e, mais recentemente, aquele da Colaboração E - 711 (AMOS et al., 1992) a $\sqrt{\mathrm{s}}=1800 \mathrm{GeV}$. O primeiro apenas incitou controvérsias desnecessárias e acreditamos que o segundo precisa ser repetido, porque os dados com erros em torno de $50 \%$, não são fisicamente aceitáveis ou significativos.

De modo particular, tomamos com objeto de análise e de crítica o caso da determinação de $\rho$ Colaboração UA4/1 (BERNARD et al., 1987) para $\overline{\mathrm{p}}$ a $\sqrt{\mathrm{s}}=546 \mathrm{GeV}$. Este é realmente um problema muito sério, porque os dados de $\mathrm{d} \sigma / \mathrm{dt}$ apresentam grandes flutuações, não penetram o suficiente $\left(-t<-t_{\text {int }}\right)$ na região da Interferência Coulombiana - Hadrônica e o ajuste não é definitivo. Os resultados mostram que se poderia ter obtido um valor de $\rho$ mais baixo apenas utilizando outras formas de parametrizações. Muitos artigos foram publicados sobre o resultado da Colaboração UA4/1, mas somente alguns tentaram mostrar que a medida não estava correta. A maioria deles procurou por explicações teóricas que justificassem ou ajustassem ao seu valor grande. E agora, o que fazer com eles?

Por fim, concluímos que para uma determinação precisa de $\rho$ a energias muito altas é extremamente necessário que os experimentos cubram com mais completeza a região da Interferência Coulombiana Hadrônica, que os dados apresentam menores erros e menos dispersões e, que os ajustes sejam realizados com parametrizações mais adequadas para a amplitude de espalhamento hadrônico elástico, diferentes da usual exponencial única. Neste trabalho, nós apresentamos duas propostas alternativas. Seria interessante que os grupos do CERN e do FERMILAB, que fazem medidas das colisões pp e $\bar{p} p$, testassem estas idéias em seus novos experimentos.

\section{Referências}

AMOS, N. et al. Measurement of small-angle antiprotonproton and proton-proton elastic scattering at the CERN Intersecting Storage Rings. Nuclear Physics B, Amterdam, n.252, p.689-714, 1985.

AMOS, N. et al. Measurement of $\rho$, the ratio of the real to the imaginary part of the $\overline{\mathrm{p}} \mathrm{p}$ forward elastic - scattering amplitude, at $\sqrt{\mathrm{s}}=1.8 \mathrm{TeV}$. Physical Review Letters, New York, v.68, n.16, p.24-33, 1992.

AUGIER, C. et al. A precise measurement of the real part of the elastic scattering amplitude at the $\mathrm{S} \overline{\mathrm{p}} \mathrm{p}$ S. Physics Letters B, Amsterdam, n.316, p.448-454, 1993.

BERNARD, D. et al. The real part of the protonantiproton elastic scattering amplitude at the centre of mass energy of $546 \mathrm{GeV}$. Physics Letters B, Amsterdam, v.198, n.4, p.583, 1987.

BLOCK, M. M.; CAHN, R. N. High energy $\bar{p} p$ and pp forward elastic scattering and total cross sections. Reviews of Moderns Physics, Minneapolis, n.57, p.563, 1985.

BOZZO, M. et al. Measurement of the proton-antiproton total and elastic cross sections at the CERN SPS COLLIDER. Physics Letters B, Amsterdam, v.147, n.4/5, p.392, 1984 .

DIAS DE DEUS, J. Minijets: elastic scattering, inelastic production and real part of the forward amplitude. New York: Goulianos, 1987.

DIAS DE DEUS, J.; PÁDUA, A. B. The search of asymptopia in soft physics at high energy. Physics Letters $B$, Amsterdam, v.317, p.428-432, 1993.

LEADER, E. Summary Talk. New York: Goulianos, 1987.

PÁDUA, A. B. et al. Uma proposta de parametrização numérica dos dados experimentais dos espalhamentos elásticos $\overline{\mathrm{p}} \mathrm{p}$ e pp a altas energias. Semina, Londrina, v.12, n. 4, p. 248-265, 1991.

PÁDUA, A. B.; PÁDUA, C. G. Teoria do espalhamento hadrônico a altas energias: aspectos gerais, aproximação eiconal e modelos geométricos.In: XXIII Águas de Lindóia 19 de outubro de 2002 